\title{
Endocytosis unplugged: multiple ways to enter the cell
}

\author{
Sudha Kumari ${ }^{1,2}$, Swetha $\mathrm{MG}^{1}$, Satyajit Mayor ${ }^{1}$ \\ ${ }^{1}$ National Centre for Biological Science (TIFR), Bellary Road, Bangalore 560 065, Karnataka, India
}

Endocytosis occurs at the cell surface and involves internalization of the plasma membrane (PM) along with its constituent membrane proteins and lipids. Endocytosis is involved in sampling of the extracellular milieu and also serves to regulate various processes initiated at the cell surface. These include nutrient uptake, signaling from cellsurface receptors, and many other processes essential for cell and tissue functioning in metazoans. It is also central to the maintenance of PM lipid and protein homeostasis. There are multiple means of internalization that operate concurrently, at the cell surface. With advancement in high-resolution visualization techniques, it is now possible to track multiple endocytic cargo at the same time, revealing a remarkable diversity of endocytic processes in a single cell. A combination of live cell imaging and efficient genetic manipulations has also aided in understanding the functional hierarchy of molecular players in these mechanisms of internalization. Here we provide an account of various endocytic routes, their mechanisms of operation and occurrence across phyla.

Keywords: endocytosis, trafficking, membrane, clathrin, dynamin, actin

Cell Research (2010) 20:256-275. doi: 10.1038/cr.2010.19; published online 2 February 2010

\section{Introduction}

Over the years, numerous modes of vesicular endocytic trafficking have been discovered that coexist in the same cell type and operate concurrently. The operation of an internalization route and its cargo specificity is determined by factors that vary in a context-dependent manner. These factors include one or more underlying principle in cargo enrichment, necessitating specific coat and coat-associated protein assembly, a scission mechanism, and a means to integrate these steps; several molecules and membrane parameters can influence and diversify an endocytic process. In this review, with the intention of summarizing present understanding of principles of endocytosis, we have surveyed different types of endocytic processes, focusing on their molecular attributes and mechanism of vesicle formation at the cell surface.

The physical and chemical properties of cargo molecules usually dictate the nature of the primary vesicular structures that participate in their internalization; the

Correspondence: Satyajit Mayor

Tel: +91-80-2366-6420; Fax: +91-80-2363-6662

E-mail: mayor@ncbs.res.in

${ }^{2}$ current address: Molecular Pathogenesis, Marty and Helen Kimmel Center for Biology and Medicine of the Skirball Institute for Biomolecular Medicine, New York University School of Medicine, New York, NY 10016 physical process of membrane deformation at initial entry is likely to dictate the mechanism necessary for endocytosis. At first glance, the scale of this initial membrane invagination provides a natural means of classifying different pathways of endocytosis (Figures 1 and 2). Ingestion of particles larger than $500 \mathrm{~nm}$ size typically occurs via triggered processes called 'phagocytosis or macropinocytosis' (Figure 1) whereas cargo below this size limit is often internalized by any of the other diverse endocytic processes available at the cell surface (Figure 2). These include the well-characterized receptor-mediated clathrin-dependent as well as the less understood panoply of clathrin-independent 'pinocytic' pathways.

\section{Macroscale endocytosis: phagocytosis and macrop- inocytosis}

There are endocytic processes that involve the internalization of large-sized particles or a large volume fraction of the extracellular bulk phase relative to the cell volume. These are termed phagocytosis and macropinocytosis, respectively (Figure 1). These processes are tightly regulated, distinct internalization events often resulting in phase-lucent structures inside cells when viewed in a light microscope [1]. They involve longrange remodeling of membrane and the cytoskeleton lying beneath it, and have been focus of some recent re- 
A

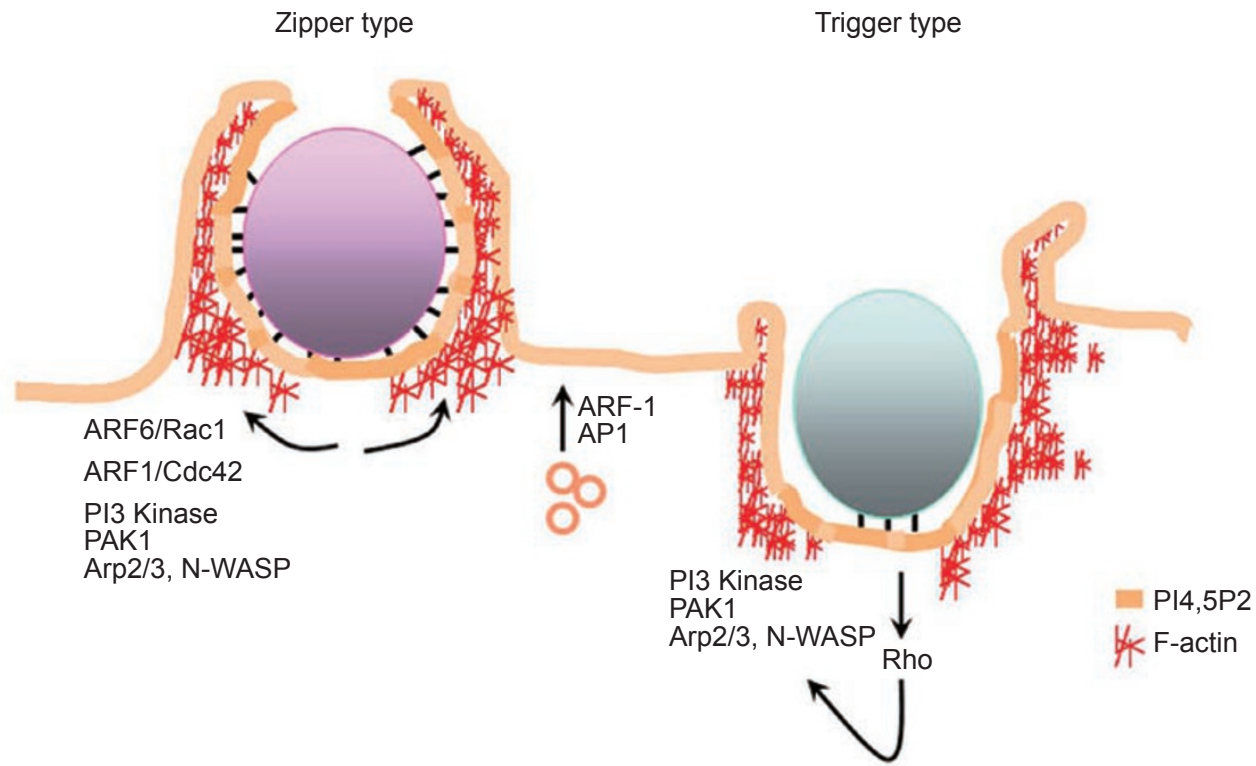

$\mathrm{B}$

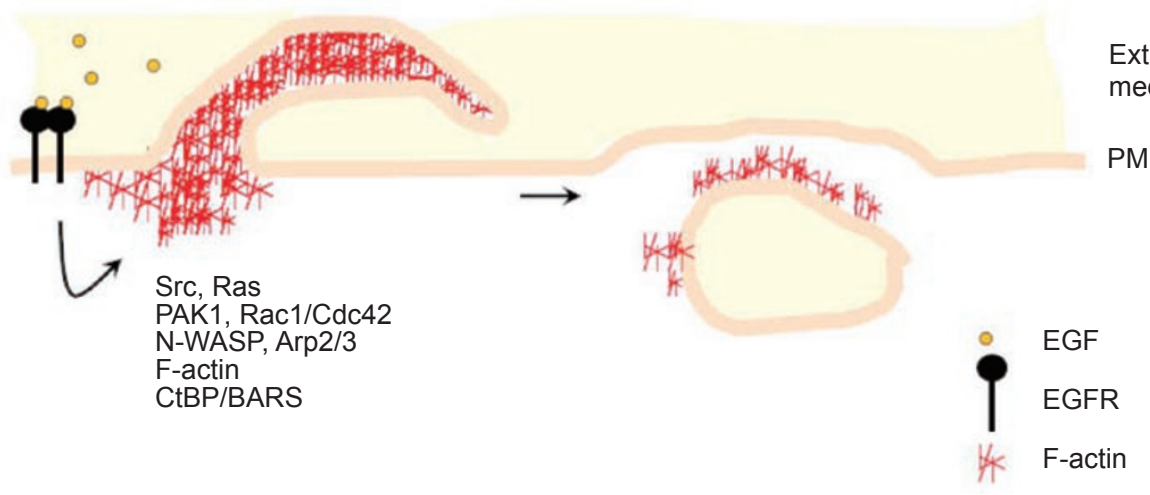

Figure1 Macroscale endocytic processes. (A) A simplified schematic representing molecular and membrane events occurring during macroscale endocytic processes. During phagocytic cup formation (left panel), sequential engagement of Fc-receptor initiates a zipper-like arrangement in opposition with bacterial surface. The process is orchestrated by stepwise involvement of GTPases and involves actin polymerization. Membrane remodeling during phagocytosis is accompanied with membrane addition to $\mathrm{PM}$, in an AP1-dependent vesicular process. In trigger-type particle entry (right panel), the process involves injection of soluble effectors into cell, which, among other events, can activate Rho GTase effectors, and hence culminating in actin polymerization at the site of bacterial entry. Variations that exhibit intermediate forms, also exist, but have not been included here. (B) EGF-induced macropinocytosis - a most extensively studied example of macropinocytosis. Binding of EGF to EGF receptor triggers the intracellular signaling cascade and activation of downstream proteins, as indicated, eventually leading to actin-rich membrane ruffle formation. Collapse of ruffle on membrane is followed with macropinosome formation.

views [1-9] and are therefore described here only briefly.

\section{Phagocytosis: ingestion of large particulates}

Classically defined, phagocytosis is a stepwise process that involves uptake of large particles into triggered cellsurface membrane deformations that usually encircle the particle, resulting in phagosomes [10]. In a metazoan system, cargoes for phagosomes range from non-selfparticles and microbial organisms to self-entities such as apoptotic cells and cellular debris. The process is initiated by cell-surface recognition of the particle, and proceeds via sequential engagement of receptors, generating 
a cup-shaped membrane distortion around the particle, culminating in a phagosome. Two well-described types of phagocytic entry are: (i) Ig receptor, FcR-mediated engulfment of immunoglobulin G-opsonized particles and (ii) complement receptor CR3-mediated ingestion of $\mathrm{C} 3 \mathrm{bi}$-coated particles. Both the means of phagocytic entry form morphologically distinct phagocytic cups and represent different types of phagocytic mechanisms (Figure 1A). Thus the morphology of forming invagination and resultant compartment is a crucial parameter for classifying these processes.

In FcR-mediated phagocytosis (termed 'zipper like'), bound FcR receptors elicit local signaling responses mediated by cytosolic immunoreceptor tyrosine-based activation motifs. This leads to localized actin rearrangement, membrane extension around ligand, and thereby further engagement of receptors with ligand in a protruding cup with zipper-lock arrangement. In CR3-type phagocytosis (termed 'trigger-like'), on the other hand, the process is triggered by treatment with either phorbol esters or extracellular agonists $[10,11]$ and the particle is loosely encased in a large membrane vesicle. The site of phagocytosis has discrete patch-like distribution of F-actin, and occurs by a regulated and triggered depression in the cell surface. There are instances, for example, engulfment of necrotic cells, where the morphology is in between the two [1]. In either case formation of phagosomes is achieved by spatiotemporally controlled sequential action of various small actin-remodeling GTPases [12]. The two modes also differ in their requirements for Rho-GTPases, in that FcR-mediated phagocytosis relies on Rac1 and Cdc42 activities, whereas CR3mediated entry is specifically dependent on RhoA $[12,13]$.

The host phagocytic machinery can also be manipulated by bacterial pathogens to facilitate their own internalization. Here too, two general models have been described that outline bacterial entry into cells: the 'trigger' or 'zipper' models. Salmonella and Shigella are examples of 'triggering' bacteria; Salmonella injects its effector, SopB, into cells, which activates an exchange factor for RhoG, leading to actin remodeling that causes internalization of the bacterium [14]. The 'zippering' model of entry of the bacteria is similar to FcR-mediated internalization, as it involves engagement of cell-surface receptors all around the particle being internalized. A good example of this 'zippering' kind of bacterium is Listeria Monocytogenes, where the bacterium interacts with host epithelial cells through its adhesins, InlA and InlB, that bind host cell receptors E-cadherin and Met, respectively [15].

Live cell imaging using RAW264.7 macrophages, a phagocyte model system, and Forster's Resonance En- ergy Transfer sensor for the small, activated GTPases, ADP ribosylation factors (ARFs) showed that FcR-mediated phagocytosis accompanies dynamic and stepwise activation of ARF6 and ARF1 [16]. This correlated with activation of Cdc42 and Rac1 [17]. Activity of the Rho GTPases is presumably required for regulation of actin polymerization during phagocytic cup formation; simultaneous inhibition of both $\mathrm{Cdc} 42$ and Rac 1 abolishes this. Inhibition of these GTPases individually however only partially inhibits phagocytosis, suggesting they act in tandem during the process. These Rho GTPases upon activation, recruit WASP and thereby the Arp $2 / 3$ complex for actin nucleation and polymerization [18]. The action of GTPases is probably regulated by the composition of polyphosphoinositides (PIPs) of the phagosomal membrane. PIPs are also intimately linked to actin dynamics [19]. During early stages of cup formation, PI4,5P2 is enriched at the inner leaflet of the cup; during phagosome closure, rapid disappearance of F-actin at the base of the cup is correlated with the reduction in local concentration of PI4,5P2. Consistent with this, overexpression of the PI-kinase (PIPKI), which converts PI4P into PI4,5P2 or inhibition of PLC and phosphatidylinositol 3-kinase (PI3K, enzymes that produce IP3, DAG, and PI3,4,5P3, respectively) abrogates phagosome closure $[2,20]$.

The shape of the phagocytic cup is usually dictated by the nature of ligand. Macrophages, when subjected to an opsonized flat surface, exhibit pseudopod extension and abortive attempts to phagocytose the surface [21]. Since the phagocytic cargo is large, sometimes as large as an apoptotic cell, internalization involves vesicular addition of membrane in the vicinity of cup. Although the origin of this membrane remains controversial, the delivery of new membrane is dependent on the adaptor protein complex, AP1, and small GTPase, ARF1 [22], suggesting a secretory origin.

The composition of phagosomal membranes has been extensively characterized. A proteomics approach helped identify more than 140 molecules present in or on latex bead-induced phagosomes in J774 cells [23]. These include, lysosomal proteins, LAMP1, LAMP2, $\operatorname{lgp} 110$, LIMPII; mitochondrial VDAC1; several members of the Ras superfamily of small GTPases, Rabs (2, 3c, 5, 7, 11, and 14); as well as proteins found in late-endosomes, alix and flotillin-1.

Once formed, phagosomes are gradually acidified and the cargo is destined for degradation. This feature of phagosomes is sometimes manipulated by pathogens, prominently by bacteria [3] to evade degradation and gain intracellular access. Further knowledge of the molecular machinery involved in phagosome formation will increase our understanding of the process as well as how 
it is manipulated by pathogens in a given context.

\section{Macropinocytosis - cell drinking}

Macropinocytosis is another process whereby a relatively large amount of the fluid phase is engulfed with respect to the cell volume, in some instances along with particles such as bacteria. First described by Lewis [6, 24] as large phase-bright organelles originating from plasma membrane (PM) ruffles, macropinosomes were initially thought to occur via non-selective membrane uptake. This view has changed with increased understanding of this process. Owing to its specific characteristics such as its inhibition with $\mathrm{Na}^{+} / \mathrm{H}^{+}$exchanger inhibitor, amiloride [25], and dependence on growth factor (GF) receptor (GFR) signaling, macropinocytosis is now defined as a highly coordinated, triggered process with distinct molecular regulators. In a study performed using soil ameba, Dictyostelium discoideum, an active phagocytic and macropinocytic organism, it was shown that during macropinosome formation, some membrane proteins were selectively depleted from the site at the PM [26]. It is now clear that there is sorting of membrane components during the formation of macropinosomes.

Macropinosomes are variable in size and can range from 0.2 to $10 \mu \mathrm{m}$ in diameter [1]. The size and morphology of macropinosomes are typically independent of ligand, although their formation can be stimulated by agents such as phorbol esters, some pathogens, and GFs [25, 27-29]. In professional antigen-presenting cells, macropinocytosis operates constitutively in quiescent circulating cells such as immature dendritic cells and is downregulated after these cells begin to mature [6, 30], suggesting a role in immune surveillance. As noted above, intracellular pathogens also exploit macropinocytosis as a means of entry into cells. Pathogens such as Shigella and Salmonella inject virulence factors that modulate the cytoskeleton, induce ruffling and subsequently, macropinocytosis. Infection rates of bacteria such as Sphingomona [31] and viruses such as HIV type I [32] are reduced upon inhibiting macropinocytosis using amiloride.

A common feature of macropinocytosis and phagocytosis is the dependence on actin-polymerization machinery. An archetypal example of GF-induced macropinocytosis is the upregulated fluid uptake in cells stimulated with epidermal growth factor (EGF) [33] (Figure 1B). Activation of EGFR signaling leads to an increase in general actin polymerization and cell ruffling. EGF binding results in activation of Rac1 GTPase as well as generation of the phosphoinositide, PI4,5P2, both of which together activate WASP/SCAR proteins. These in turn bind to the Arp2/3 complex, hence modulating actin po- lymerization. Most instances of macropinocytosis, with few exceptions (for example, bone marrow dendritic cells, where Cdc42 is implicated [34]), are dependent on Rac 1 activation).

Another molecular regulator of macropinosome formation is PAK1 (p21-activated kinase); manipulation of PAK1 activity correlates with macropinocytic extent in NIH3T3 cells [35]. While PAK1 is capable of phosphorylating and therefore activating $\mathrm{Cdc} 42 / \mathrm{Rac} 1$, it also phosphorylates CtBP1/BARS proteins. CtBP1/BARS (C-terminal-binding protein-1/brefeldin A ribosylation substrate) proteins were originally demonstrated to regulate dynamin-independent fluid uptake in a variety of cell lines, and were later reported to localize to the site of and affect macropinosome membrane closure in a phosphorylation-dependent manner [36]. It is possible that BARS proteins represent a link between dynamin-independent fluid endocytosis and macropinocytosis (see below).

The nature of fission machinery involved in macropinosome scission from the PM is not yet clear. There is evidence that implicates isoforms of dynamin in specific contexts. Although EGF or serum-repletion-induced macropinocytosis in epithelial cell lines is insensitive to dynamin inhibition [37], macropinocytosis is severely impaired in PDGF-stimulated dynamin-null embryonic stem cells [38]. In these embryonic stem cells, fluidphase tracer uptake, as an indicator of stimulated macropinocytosis, was rescued by reintroduction of dynamin-1 or 2 [38], suggesting that dynamin is crucial for this form of macropinocytosis. However, at this stage it is not clear whether dynamin could serve as a scissoring protein for specific cases of macropinocytosis, or has other regulatory functions for endocytosis. Dynamin is known to regulate cellular distribution of Rac1 [39], and therefore can indirectly influence macropinocytosis by regulating the availability of Rac1, necessary for actin remodeling.

Even though phagocytosis and macropinocytosis differ in their nature of induction and detailed mechanisms, these processes share multiple operational similarities, pointing toward similar membrane remodeling pathways during internalization. Both have slow kinetics, which incidentally enables the study of the formation of these vesicles in live cells. Multiple studies have demonstrated localization of regulatory proteins to phagocytic cups and macropinosomes $[16,36]$ as well as the local and distinctive membrane composition during their formation [40]. Both processes employ molecules such as the PI3K and other actin polymerization effectors, and are typically initiated by phosphoinositide-dependent processes. Since dependence for endosome formation on the actin machinery is also exhibited by many other endocytic pathways, the distinctive feature of phagocytosis and 
macropinocytosis still remains their large size.

\section{Microscale endocytic processes}

Endocytosis at a scale smaller than $200 \mathrm{~nm}$ poses specific demands on membrane machinery needed to bend membrane at this scale [41]. The eukaryotic cell appears to have resolved this in many ways. There are several modes of endocytic processes, distinguished by specific sets of molecular regulators and functional modules that are associated with their optimal operation. Molecules involved in the generation of a vesicle can be part of a module assisting coat assembly, or involved in the pinching process, or may represent specific membrane lipids and lipid-modifying proteins. Here we discuss these attributes and the endocytic processes that have come to be defined by the combinations of them. A convenient way of classifying some of the modes of microscale endocytosis is by the nature of the coat proteins that are associated with a specific process (Table 1, Figure 2).

\section{Coat protein-mediated pathways}

Clathrin-mediated endocytosis Initially identified in electron micrographs used to study yolk protein uptake in mosquito Aedes aegypti, clathrin was one of the first endocytic coat proteins to be discovered as a major component of 'bristled vesicles' $[42,43]$. Studies on clathrinmediated endocytosis have dominated and defined the paradigm for endocytosis. Morphological studies with clathrin indicated that it is capable of coating vesicles $100-200 \mathrm{~nm}$ in diameter. Clathrin functions as a trimer of heterodimers, each unit consisting of one heavy and one light chain forming a triskeleton [44]. These triskelia can assemble into a lattice-like structure around the vesicles. Adaptor proteins function to link up specific cargo with the clathrin coat [45]. Simply put, clathrin-pit-mediated endocytosis involves cargo recognition and coat assembly, followed by membrane invagination, and finally pinching off of the dimpled deformation.

Although it was believed for a long time that clathrinmediated endocytosis is a cargo-induced process, it is now known that clathrin coats can spontaneously assemble at the PM and are stabilized by interactions with cargo [46]; the presence of sorting motifs YXXF, DEXXXLLI, FXNPXY, and polyubiquitination in transmembrane proteins that can bind to adaptor proteins often couple productive coat formation to capture of cargo [47].

Clathrin-coated vesicle (CCV) formation and its regulation have been reported to be associated with $\sim 150$ proteins, however, at this stage, with the exception of a few, their precise sequence of action during CCV formation remains unexplored. In a study carried out in live

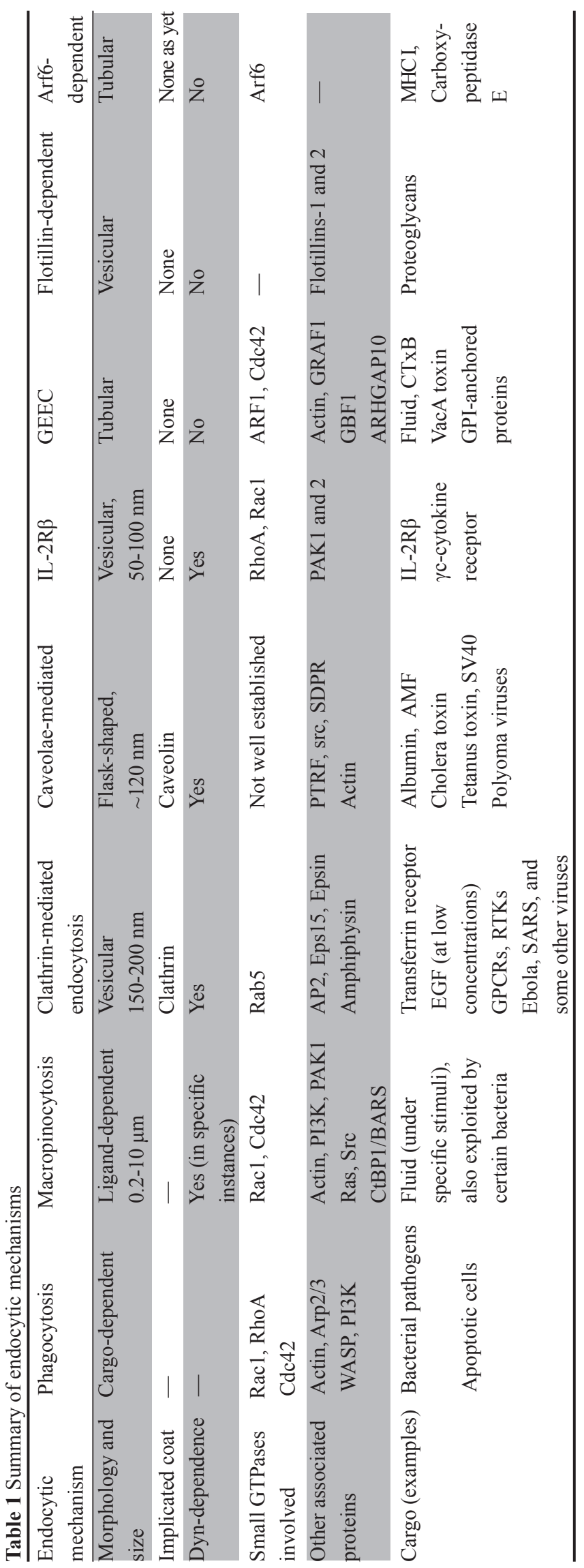


A

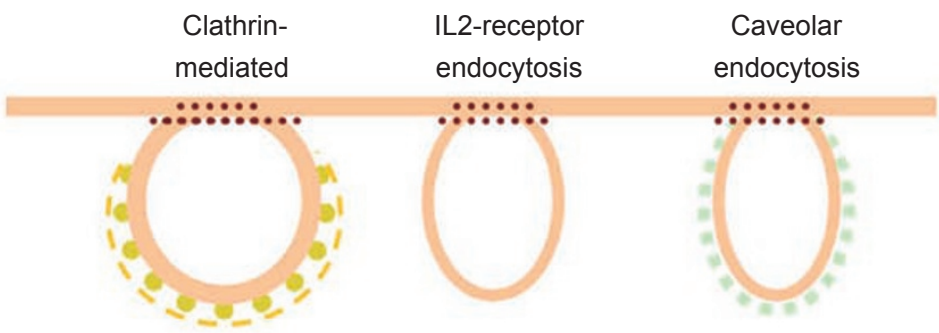

Receptor sorting motifs

Rho A

Receptor sorting motlfs

Adaptors and associated proteins

Rac1

Caveolar coat cholesterol

Clathrin lattice

Dynamin

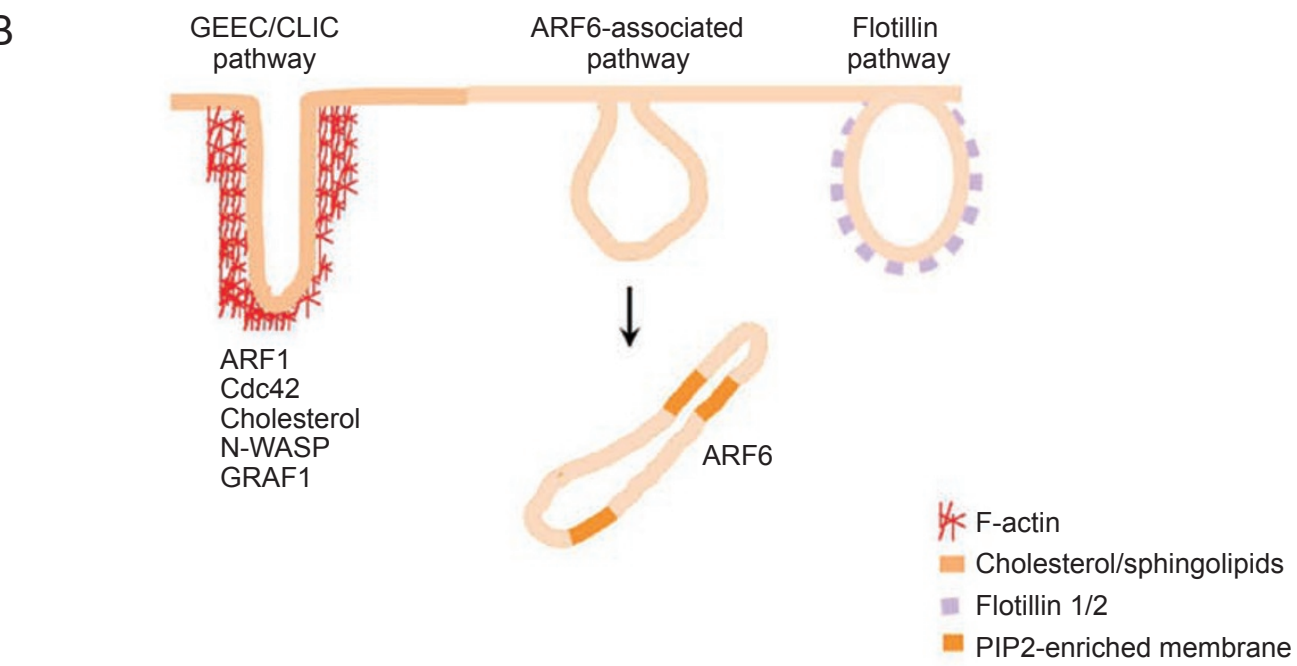

Figure 2 Microscale endocytic processes. Microscale endocytic processes can be broadly classified according to the requirement for dynamin for pinching. (A) Pathways that are dependent on dynamin activity for vesicle formation, (B) Pathways that are independent of dynamin function. Most examples in (B) are less-explored in terms of their molecular mechanisms, and their specificity for particular cargo is less well defined. For example, CD59 is endocytosed by both the ARF6-associated pathway and the flotillin-mediated endocytic route. The routes illustrated here represent pathways that have been shown to be distinct from one another.

cells using clathrin-GFP and TIR-FM, Merrifield et al. [48] uncovered the chronology of a few effector protein dynamics during the formation of a mature coat. More of such investigations will shed light on dynamics of critical molecular events that occur during CCV formation.

Imaging of clathrin-coat nucleation at PM suggests that it assembles randomly, mediated by cargo protein,
AP, and accessory proteins [46]. In neurons, APs direct lattice formation to specific sites at PM via interaction with PI4,5P2 and synaptotagmin I and clathrin. This assembly can be further stabilized by epsin and AP180/ CALM, which bind to AP2, PIP2, and clathrin [49]. Subsequent to this, stabilization of the clathrin-coated pit (CCP) requires cargo acquisition via subunits of AP2 and 
clathrin $[46,50]$. This step proceeds via the recruitment of various AP2-binding partners that facilitate cargo binding, optimal clathrin polymerization, and membrane bending. The process of membrane bending is brought about in part by BAR (Bin Amphiphysin Rvs) domaincontaining proteins [9], such as endophilin and amphiphysin, and is further facilitated by epsin and inherent curvature properties of assembled clathrin structures [51, 52]. Mature clathrin-coated vesicle, while still attached to the PM, contains amphiphysin and recruits the pinching module that contains the protein dynamin. Dynamin eventually promotes the scission of the vesicle (see later section for details).

The prevalent view of clathrin assembly is of a homogenous, symmetric polygonal lattice [53], the size of individual vesicle varying depending on the cell type of origin. CCPs at the cell surface are of diverse composition with respect to the usage of adaptor and associated proteins $[54,55]$, and offer distinct microenvironments for the regulated entry of specific combinations of cargoes [56]. Interestingly, ligand concentration also affects the mode of this endocytic route: for example, EGF, at low concentrations, is internalized by a clathrin-dependent endocytic route [57], but at higher concentrations, seems to enter cells through clathrin-independent routes with consequences for signaling via the EGFR [58]. How such fine-tuning and discrimination are regulated is a subject of current investigation [56].

Several viral pathogens such as Ebola virus, SARS coronavirus, and some nonenveloped mammalian reoviruses enter cells by receptor-mediated endocytosis, targeting receptors internalized by the clathrin-dependent pathway [59]. For some viruses this route is obligatory, and for others the clathrin pathway is one of a few that support infection. Studies on the entry of influenza virus in epithelial cells have indicated that a large fraction is endocytosed into CCPs; viral particles are also endocytosed by non-clathrin pathway operating in parallel [60]. Viruses that take a clathrin-dependent route more often induce formation of clathrin pit at the site of binding at the PM rather than entering a preassembled clathrin structure [59]. This suggests a flexibility in regulation of clathrin-coated vesicle formation. Similarly, in case of bacterial uptake, the entry of the bacterium, L. monocytogenes, into HeLa cells was sensitive to depletion of clathrin heavy chain and other endocytic proteins such as dynamin, Eps15, but not the adaptor AP2. This was unexpected because it suggests that cargo 20 times larger than a normal endocytic cargo could use the clathrinbased machinery to enter a non-phagocytic mammalian cell, reiterating the flexibility in clathrin-mediated endocytic processes, both in terms of size and associated machinery
[61].

Caveolin and the caveolar endocytic process Another membrane coat at the cell surface is caveolin. Caveolae were originally described as flask-shaped structures in ultrastructure of blood capillaries by Palade in 1953 [62], but it was not until much later that the coat component, caveolin, was identified [63]. Caveolae are formed by assembly of caveolins, integral membrane proteins that bind directly to membrane cholesterol. There are three subtypes of caveolin proteins, caveolin-1, caveolin-2, and caveolin-3, the former two being responsible for caveolae formation in non-muscle cells and latter in muscle cells [64]. A recent study demonstrated that while morphological features of caveolae are widely conserved, the functions of individual caveolin proteins may be diverse [65]. Many mammalian cell lines such as human colorectal adenocarcinoma cells, Caco2 [66], or tissues (e.g. many blood cell lineages) do not have detectable levels of caveolin, pointing toward the specialized tissuespecific function of caveolins. Overexpression of caveolins in the cells lacking them induces de novo formation of caveolae [67, 68].

The precise function and regulation of caveolar assembly have been a matter of intense investigation. Caveolins have a predicted hydrophobic stretch of potential hairpin structure composed of $\alpha$-helices. Insertion of transmembrane amphipathic helices in a wedge-like manner such that inner leaflet lipids are displaced more than outer leaflet lipids could induce membrane curvature [69]. Moreover, caveolins are capable of oligomerization (caveolin-1 and caveolin-2) on membranes, which could further potentiate membrane curvature. Consistent with this idea, the density and number of caveolae generally correlate with cellular caveolin expression [70]. Caveolae formation is cholesterol dependent and loss in membrane cholesterol leads to disassembly of the caveolar structures.

Recent investigations have identified additional factors for the coat component of caveolae, polymerase I and transcript release factor (PTRF), and serum deprivation protein response (SDPR) $[8,65]$. These molecules appear to be both part of the coat and necessary for the assembly of caveolae from distributed caveolins at the PM. Loss of PTRF in mammalian cells as well as in zebrafish correlates with lack of caveolar structures [71]; PTRF possibly acts by modulating relative amounts of caveolae-bound caveolin to free caveolin in the PM [71], whereas SDPR is likely to be a protein that is required at the sites of caveolar assembly.

The caveolar pathway is responsible for the endocytosis of ligands such as albumin [72], autocrine motility factor [73], tetanus toxin [74], cholera toxin [75], and 
viruses such as polyoma and simian 40 (SV40) $[76,77]$; to name a few. In many of these cases the receptors for specific cargoes have also been identified but the mechanism by which cargo is concentrated in caveolae is not understood. In addition, endocytosis via caveolae also remains poorly characterized. It is not clear if caveolar endocytosis is constitutive, albeit occurring at a low rate, and is massively up-regulated on specific induction. Fluorescence recovery after photobleaching studies has indicated caveolae to be relatively stable structures on PM [78], which can however be made to bud off by the interaction of pathogens such as SV40 virus [79] or can also be induced by incorporation of fluorescent analogues of sphingolipids, namely, lactosyl ceramide [80-82]. During integrin-dependent cell adhesion, deadherence induces removal of caveolin-coated membrane from the cell surface, and it has been shown that caveolin is required for this process [83]. This results in altered PM lipid composition, due to the loss of many lipids and proteins that partition into detergent-resistant membrane (DRM) domains. This has significant functional consequences foremost of which is loss of Racl activity at the cell surface, and consequently remodeling of the cortical cytoskeleton, and activation of anoikisis, a form of apoptosis induced by the loss of cell anchorage [83, 84].

Caveolin knockout mice have proven to be useful genetic tools for studying the function of caveolin and ascertaining caveolar cargo specificity [85]. In mouse embryonic fibroblasts (MEFs) obtained from caveolin-1 knockout mice, albumin uptake was found to be defective [86], but the entry of SV40 [87] and cholera toxin B subunit (CTxB) [88], two typical cargoes of caveolae, was not compromised. This could be explained by the possibility that caveolin, when present, induces formation of caveolae, but is dispensable for internalization of some caveolar cargoes. In addition, there is evidence that caveolins can negatively regulate entry of certain cellsurface proteins, including CTxB [89, 90], where overexpression of caveolins prevents their internalization, suggesting that apart from their direct role in endocytosis, caveolins can indirectly influence other endocytic pathways. A novel role for caveolin-1 has been reported, in regulation of Cdc42 activity during membrane trafficking events. Caveolin-1 binds preferentially to $\mathrm{Cdc} 42$ in its GDP-bound form and functions as a guanine nucleotide dissociation inhibitor (GDI) for Cdc42. Thus, by maintaining Cdc42 in an inactive state, caveolin-1 regulates basal secretion in the absence of stimuli in pancreatic $\beta$-cells [91]. It is possible that caveolin- 1 has similar indirect regulatory roles at PM via inhibitory interactions with mediators of other endocytic pathways. Endocytosis of SV40 virus has been reported to increase in cells lacking caveolin-1 [87], indicating an inhibitory role for caveolin-1 in this process. However in this study it was not clear whether this effect is due to squelching of effector molecules or due to sequestration of a specific kind of membrane components. Studies exploring redundant endocytic roles of caveolin will be invaluable in understanding the regulatory cross-talk between caveolar and non-caveolar internalization pathways.

\section{Dynamin-mediated endocytic vesicle scission}

Once a coated patch of membrane has been generated, the formation of an endocytic vesicle requires fission of the budded membrane from the parent membrane. A large GTPase, dynamin, has been implicated in multiple fission processes in eukaryotic cells [92-94]. There are three dynamin genes identified in mammalian cells, each gene expressing at least four multiply spliced forms (isoforms). Dyn1 is restricted to neuronal cells; $D y n 2$ is ubiquitously expressed; and Dyn 3 may be limited to brain, lung, and testis tissues [95].

A wide variety of endocytic processes, including clathrin-dependent receptor-mediated endocytosis, require dynamin for the scission of endocytic intermediates to generate vesicles (Figure 2A). Originally, dynamin was identified as a microtubule-binding protein and was found to be homologous to the Drosophila shibire gene $[96,97]$, mutations in which were shown to cause a temperature-sensitive paralysis. Insights into dynamin function came primarily from morphological analysis of synapses in the shibire ${ }^{\text {ts } 1}$ mutant. Cycling of mutants through restrictive and permissive temperatures, Koenig and Ikeda observed a reversible loss of synaptic vesicles at neuromuscular synaptic junctions, implicating dynamin in synaptic vesicle endocytosis [16]. Overexpression of dominant negative, GTP-binding mutants of dynamin also blocked receptor-mediated endocytosis in various cells, suggesting a role for the GTPase activity of dynamin in the clathrin-dependent endocytic process outside the nervous system. Dynamin was found to localize to CCPs at the PM and in isolated synaptosomal preparation $[98,99]$, providing further evidence of a direct role for dynamin in endocytosis.

Dynamin is likely to be recruited during clathrin-pit assembly, a process where N-BAR domain-containing proteins, such as amphiphysin, which can directly interact with dynamin [100], are also recruited. At the molecular level, dynamins contain a Pleckstrin homology (PH) domain, a GTPase effector domain (GED), and a Cterminal proline/arginine-rich domain (PRD). GED can interact with the GTPase domain of an adjacent dynamin molecule thus causing oligomerization of the protein [101], and concomitant activation of the GTPase activity. 
The PRD of dynamin can interact with a variety of proteins, including the ones that contain Src homology SH3 domain, and $\mathrm{PH}$ domain binds to $\mathrm{PI} 4,5 \mathrm{P} 2$ phosphoinositides. Wide range of interaction partners for dynamin enables it to recruit and bind proteins during coated vesicle formation.

At high local concentrations, dynamin is also capable of forming multimers and spirals on membranes. Dynamin oligomerization and dynamics lead to constriction and eventually budding of the vesicle. The exact function of dynamin at the level of vesicle fission has been a subject of controversy. There are various hypotheses regarding the GTP-dependent mechanism of dynamin action for vesicle pinching. First is the conformational change in dynamin brought about by GTP loading (switch model), which can cause scission by recruiting other molecules that will fuse membranes and acts essentially as a timer for this reaction, whereas the other relies on the actual mechanical force generated at the vesicle neck (pinchase model). While there is evidence for both the GTP switch and mechanoenzyme functions of dynamin [102], recent studies support a mechanoenzyme or motor-like function of dynamin. The in vitro demonstration of dynamin-coated lipid tubules twisting in response to supply of GTP was a direct evidence supporting a mechano-enzyme behavior where the authors argue that in addition to membrane tension, torsional strain generated at the neck of the vesicle can lead to pinching [103]. Two other studies demonstrated that it is the kinetics of dynamin depolymerization and polymerization on 'tension-free' membranes that brings about changes in membrane curvature, necessary for fission $[104,105]$. At this point both these possibilities have distinct implications on the dynamics of dynamin action during pinching as discussed [106].

Apart from acting as a part of the scission machinery for the clathrin-dependent pathway, dynamin function is also implicated in vesicle formation in a set of clathrinindependent pathways, including caveolar endocytosis [107] and a class of macropinocytosis [38, 108]. Other clathrin-independent pathways that utilize dynamin are the RhoA-dependent IL-2 receptor endocytic route [109] and APP endocytosis in primary neurons [110]. Although ultrastructural studies indicate that IL-2 receptors are not recruited to CCPs, they were internalized by small uniform-sized invaginations. The uniform and small size $(50-100 \mathrm{~nm})$ of the forming endocytic pit at the surface that appears to concentrate the cargo suggests the presence of a protein coat that could aid in the recruitment of these proteins to the site of endocytosis. A lot more information is necessary to uncover the mechanism of operation of this type of endocytosis.

Similarly, there exists an unusual mode of endocytic clearance of EGFR from dorsal surface of migratory epithelial cells. Here, cells on stimulation with EGF generate circular wave-like ruffles that on concentric closure internalize $\sim 50 \%$ of cell-surface EGFR. This process employs dynamin 2 but is independent of clathrin or caveolins [111]. Overall, the function of dynamin seems neither to be strictly dependent on the presence of clathrin on buds, nor is limited by the size of invaginations. As indicated above, the mechanism by which dynamin is recruited to these endocytic sites at PM is unclear, and ought to be the subject of further investigations. However, a general theme that emerges is that most coat-dependent mechanisms of endocytosis use dynamin to assist in scission of the membrane deformation from the cell surface. On the other hand there are a number of coatindependent endocytic processes that appear to function in the absence of dynamin (Table 1).

\section{Clathrin- and dynamin-indepenent endocytosis}

At this time there are a number of endocytic pathways where neither specific coat proteins nor a particular pinching system have been identified (Figure 2B). In fact, the existence of a coat-independent route for endocytic uptake has taken a long time to be well accepted; careful experimentation coupled with genetic manipulations provided the necessary evidence. For example, the acceptance of clathrin-independent endocytosis was built on a number of studies: (i) injection of African green monkey kidney cells (CV1) with anti-clathrin antibodies abolished receptor-mediated uptake completely, but inhibited the uptake of a bulk phase marker (lucifer yellow) by only $40 \%$ [112], and (ii) clathrin-dependent endocytosis could also be inhibited by hypotonic shock, cytosolic potassium depletion, or acidification of cytosol while under all these conditions, endocytosis of the plant toxin ricin continued to take place $[113,114]$. Together, these and other experiments paved the way to the idea that cells could exhibit one or more clathrin-independent endocytic routes.

In some instances, ultrastructural visualization of endocytic intermediates did not reveal electron-dense coats [88], suggesting that either these pathways do not have a proteinaceous coat or the coat assembly, if any, is too transient to be captured by current methodologies of cell fixation and processing. It is also possible, however, that lipid or protein accretions could initiate membrane deformation (vesicle formation) without defined coat proteins. While the pathways that require coats also have their specific lipid requirements $[115,116]$, in some instances, lipid organization alone has proven to be sufficient for membrane invagination. Shiga toxin entry is one such example where binding of toxin to the ganglioside, $\mathrm{Gb} 3$, 
induces invaginations in cells as well as model membranes [117]. This was specific to toxin binding, since simply cross-linking Gb3 with antibodies failed to result in invaginations. These studies provide evidence for the requirement of a specific lipid-binding architecture for this process. A lipid-compaction model was proposed for the segregation and membrane budding induced by the toxin. This process was inhibited by increasing membrane tension, indicating that membrane properties, tension, and bending rigidity might play a crucial role in the regulation of lipid-based invaginations. Endocytosis of the lipid-anchored proteins such as glycosylphosphatidylinositol-anchored proteins (GPI-APs) also does not appear to require any of the well-characterized coat proteins $[64,88,118,119]$. This pathway could be another example where the foundation for membrane deformation is laid by membrane lipids (see the CLIC/GEEC pathway below). An example where a protein platform suffices to support endocytosis is the process of human papillomavirus 16 (HPV16) virus infection, where tetraspanin-enriched domains are required for virus entry [120].

It was widely accepted that dynamin would be indispensable for all endocytic activities in the cell. In part this notion had been fuelled by the existence of a number of dynamin genes, and isoforms/splice variants [95, 121, 122]. However, this notion was difficult to reconcile with a few key observations: (i) overexpression of dominantnegative mutant form of dynamin-2 in HeLa cells [98] inhibited clathrin-dependent endocytosis but could not completely block fluid phase uptake, and (ii) hemocytes derived from the temperature-sensitive shibire mutants of Drosophila exhibited a complete block in clathrindependent endocytosis of scavenger receptors, whereas fluid-phase and GPI-AP uptake remained unaffected [123]. The dynamin gene mutation in Drosophila renders all dynamin splice forms mutant since the mutation lies in the common exon encoding the GTPase domain [124].

To assess a role for dynamin in a pathway, most studies have used dominant-negative inhibition of dynamin to test concomitant quantitative defects in endocytic cargo uptake. Interpretation of such experiments is however, complex. It is difficult to ascertain whether lack of defect is due to lack of involvement of dynamin, or due to an alternative internalization pathway that is induced by inhibition of dynamin. Alternatively, the ability of dynamin mutants to interfere with the activity of Rac [39] suggests that inhibition of a particular pathway may not reflect a direct role for dynamin. From the existing examples under the canopy of dynamin-independent pathways, there are also specific requirements for small GTPases such as the ARF family proteins, and the Rho family proteins (Table 1). These GTPases could function as timers for regulation of the assembly and disassembly of actin-based endocytic mechanism or as scaffold to recruit other regulatory proteins. However, it is evident that while these GTPases can be utilized in combination, they have a diversity of functions not solely restricted to endocytosis [109, 125].

\section{Endocytosis mediated by the actin cytoskeleton}

There is a growing understanding of the molecular mechanism involved in the operation of different endocytic pathways and the role for the actin cytoskeleton in its regulation [126] (see Table 1 and the following sections). This necessitates a new understanding of the role that actin and its associated molecular network may play in different aspects of the endocytic process. Actinmeshwork could function as a coat and/or help in the recruitment of adaptor proteins, thus allowing for cargo concentration and membrane deformation, leading to the formation of membrane buds. In addition, coupled with motor protein activity and dynamic polymerization, it could play a role in the generation of a scission force.

In this context, extensive genetic, biochemical, and morphological studies in budding yeast have provided valuable insights. In a mutant screen for endocytic defects in yeast, a large number of identified genes were related to actin and its regulators [127]; subsequently, the recruitment of actin to the sites of endocytosis was elegantly demonstrated in live cells [128]. One of the first identified genes involved in $\alpha$-factor uptake was act 1 (end7), the yeast actin gene. Nearly a third of endocytic proteins identified in yeast bind actin or regulate its assembly. A specific kind of actin structure, called 'cortical actin patches' has been observed to be associated with endocytic sites. Using real-time fluorescence microscopy and particle tracking, Drubin and colleagues [129] demonstrated that there are different cortical actin patches with distinct protein composition and motility properties; and these were intermediates in the endocytic process. Subsequently, using a live imaging screen of deletion mutants, where they dissected defects in coat dynamics and actin assembly, they provided evidence for the existence of distinct protein modules for coat formation, membrane invagination, actin-meshwork assembly, and vesicle scission during clathrin- or actin-mediated endocytosis [130]. In the same study, they also demonstrated that, in yeast, while clathrin facilitates endocytic site assembly, it is not required for membrane invagination or vesicle formation. Based on these studies, a fission mechanism for such a pathway has been recently proposed [131]. This model suggests that endocytosis can be initiated at a site where the membrane invaginates, and then elongates into a tube through the encasement by ac- 
tive polymerization of actin, culminating in the scission of a membrane bud of different membrane and protein compositions via traction by myosin motors [132]. Considering that clathrin and its effectors also associate with these vesicles, it is not yet established if actin alone acts as the coat [133].

In mammalian cells, specifically NIH3T3 cells, actin and cortactin are asymmetrically associated with highly dynamic early endocytic structures and move correlatively with these vesicles, supporting the notion that actin could generate the force required for vesicle movement and thus fission [134]. Disruption of the actin cytoskeleton also causes a reduction in endocytosis in a number of clathrin-independent pathways [64, 135]. Large vesicles formed as a result of compensatory endocytosis in $\mathrm{Xe}$ nopus oocytes also have been shown to have actin coats around them, although the precise function of actin there has not been addressed [136]. It should be, however, noted that actin is a multifunctional structural protein and perturbation in actin and actin nanomachinery can also result in non-specific and indirect defects in endocytosis; nevertheless colocalization of F-actin with endosomes indicates a more direct role in endocytosis. In the following sections we discuss some of the pathways that utilize actin and its regulators, in further detail.

The CLIC/GEEC pathway As discussed above, endocytosis of GPI-APs represents a clathrin- and dynamin-independent internalization route. GPI-APs are outer leaflet membrane proteins that lack cytosolic extensions and thus are unable to directly bind cytosolic adaptors. These proteins belong to a family of proteins with diverse functions and have been shown to be internalized by a dynamin-independent endocytic route, into a specialized early endosomal compartment, where a majority of the internalized fluid phase is also detected in many cell types [119]. Endocytic structures containing nascent endocytosed GPI-APs are named GPI-AP enriched early endosomal compartments (GEECs). GEECs are proposed to result from fusion of uncoated tubulovesicular CLICs (clathrin-independent carriers) directly derived from the cell surface [88].

CLICs/GEECs are selectively enriched for GPI-APs [119] although at this stage the sorting determinants at PM are not clear. It could potentially be lipid-based sorting, as alterations in cholesterol and sphingolipid levels as well as perturbation of the nanocluster state of the GPI-AP affect endocytosis through this pathway [64, $119,137]$. However, a recent study shows the size of extracellular moiety attached to the lipid tail as an additional determinant for inclusion into GEECs [138]. It will be interesting to investigate the size range of proteins that allows them to be endocytosed via GEECs and also, how the size-based regulation crosstalks to lipidmediated sorting into the GEEC pathway. Another feature of endocytosis via CLICs/GEECs is its dependence on the actin polymerization machinery [64]; maintenance of dynamic cortical actin architecture appears to be required for GEEC endocytosis. This is brought about by the cycling of the Rho family GTPase Cdc42 and downstream activation of the WASP protein, since locking of Cdc42 in GDP or the GTP state leads to inhibition of the GEEC pathway [135]. Cdc42 dynamics on PM is regulated by ARF1; activation of ARF1 leads to recruitment of a Rho-GAP domain-containing protein, ARHGAP10, and inactivates $\mathrm{Cdc} 42$, thus maintaining its cycling [135]. Another recently reported effector of GEEC formation is a BAR domain-containing protein, GTPase regulator associated with focal adhesion kinase-1 (GRAF1). Endogenous GRAF1 localizes to CLIC/GEEC carriers in HeLa cells and silencing its expression results in reduced GEEC marker uptake [139]. GRAF1 colocalizes with active Cdc42 and contains a Rho-GAP domain, so it may be involved in regulating $\mathrm{Cdc} 42$ dynamics apart from its other functions. GRAF1 also contains a BAR domain, which could aid in membrane curvature and an SH3 domain, capable of interacting with dynamin GTPase. It is interesting to note that while GEEC formation is dynamin-independent, dynamin appears to be recruited to GEECs post internalization [88]; this could potentially be mediated by GEEC-associated proteins such as GRAF1, which has been shown to interact with dynamin.

Although there is increasing information about the molecular regulation of GEECs, the functional relevance of this pathway remains unexplored. Since a variety of GPI-APs are internalized by the GEEC pathway, and in some cases endocytosis via GEECs specifically aids in accomplishing their cellular function [140], this pathway could be of vital importance for this whole family of proteins. Considering its constitutive nature, fast kinetics and heterogeneous resultant vesicle size, the GEEC pathway could mediate the regulation of PM homeostasis. There are an increasing number of proteins internalized by pathways that bear a resemblance to the GEECs. Endocytosis of cholera toxin [88], internalization of VacA toxin, and sorting nexin 9 (SNX9)-regulated GPIAP internalization routes are examples similar to GEECs $[141,142]$. Another cargo for a GEEC-like pathway is dysferlin, a muscle repair protein, mutations in which are associated with several myopathies. In MEFs devoid of caveolin-1, dysferlin is endocytosed into compartments containing GPI-APs and its cellular entry is independent of dynamin activity, indicating that internalization could potentially be carried out via the GEEC pathway [89].

ARF6-dependent pathway Cell-surface proteins such 
as MHC I and Tac (the IL-2 receptor $\alpha$-subunit) are internalized in a dynamin (and clathrin)-independent manner into an ARF6-positive tubular endosomal system. These are distinct from the clathrin-dependent cargo-containing endosomes [143]. Overexpression of constitutively active form of ARF6 was shown to increase endocytosis of Tac; however, inhibition of ARF6 via overexpression of a GTP-exchange defective form does not perturb endocytosis [143]. There have been several additions to the cargoes and effectors of this pathway, recently reviewed [144].

Endosomes-containing cargo internalized via this pathway, remain associated with activated ARF6 [145]; hyperactivation of ARF6 traps a PIP2-enriched early endosomes of a vacuolated morphology, while its constitutive inactivation blocks the process of recycling back to PM from tubular compartments. Recently, candidates for cargoes endocytosed via the ARF6-pathway were identified using hyperactivated ARF6-trapped endosomes [146]. While ARF6 associates with endosomes containing a specific set of cargoes, it is not clear whether it is involved directly at the internalization level or the recycling step or both. The localization of ARF6 on endosomes can result both from stable association during endocytosis and acquisition on endosomal membrane post-internalization. While ARF6 regulates dynamindependent endocytosis of the herpes simplex protein vp22 [147], it has also been demonstrated to control the recycling of membrane [148]. It will of interest to visualize the localization of ARF6 in the context of cargo endocytosis at PMA.

Although the ARF6 pathway bears a resemblance to the GEEC pathway, it is likely that these two processes are distinct modes of endocytic trafficking. Perturbation of GRAF1 in HeLa cells causes inhibition of fluid uptake (a GEEC marker which is internalized into tubular endosomes as well), but it neither affects MHC I internalization nor does it associate with MHC I-positive structures [139]. An important function of this pathway may be to regulate membrane availability for migratory cells, and recycle activated small GTPases to the PM [149]. It is also responsible for recycling of a prohormone sorting receptor, carboxypeptidase E, to the Golgi [150].

\section{Other dynamin-independent endocytic pathways}

Role of flotillins Flotillins appear to outline a dynaminand clathrin-independent endocytic process, since cargoes including fluid phase and a GPI-AP, CD59, in HeLa cells are endocytosed via this pathway [151]. In the cells depleted of flotillin-1, there is a defect in uptake of GPI anchor protein CD59, and CTxB internalization is shifted to a dynamin-dependent mode. However, the relationship of this pathway to the GEEC/CLIC pathway in other cell types is not yet established; a role for cholesterol and specific RhoGTPases remains as yet unaddressed.

There are two flotillin genes, flotillin-1 and flotillin-2 (identical to reggie-2 and 1, respectively) [152]. In live cells, flotillins are localized to small puncta. Flotillin-1 and 2 are both required for induction of membrane invaginations in a dose-dependent manner [153]. Similar to the caveolins, flotillins are associated with DRMs [23]. While flotillins were shown to form heteromeric complexes with caveolins, as demonstrated by co-immunoprecipitation experiments, it is yet unknown if this interaction is functional in the context of caveolae formation [154]. In MEFs lacking caveolin-1, while majority of flask-shaped invaginations are absent, there are some structures that remain. This suggests a role for other molecules in the formation of caveolae (flask)-shaped invaginations [155]. By EM and immunogold labeling, flotillin appears to decorate small membrane invaginations distinct from clathrin- or caveolin-1-labeled pits. However, in a recent study in fibroblasts lacking caveolin-1, overexpression of flotillin isoforms, flotillin-1 and 2 could not induce flask-shaped invaginations, suggesting that factors more than flotillins may be necessary for the generation of the flotillin-decorated invaginations [156].

A recent study has implicated flotillin-1 in CXCR4 recruitment to DRMs and signaling [157]. It is possible that flotillins generate specialized surface platforms that aid in the regulation of signaling of immunoreceptors and their endocytosis. The trigger (if any) for flotillin-mediated endocytosis has recently been shown to be initiated by Fyn-mediated phosphorylation, suggesting that this endocytic process is regulated by non-receptor tyrosine kinase activation [158]. In summary, while flotillins may define a dynamin-independent endocytic process, the studies thus far raise interesting questions regarding the function of endogenous flotillins, their means of interacting with specific cargo and the mechanism of vesicle generation.

Role of tetraspanins Another protein platform that supports endocytosis is the family of tetraspanins. Dynamin-independent endocytosis of HPV16 is localized to tetraspanin domains (CD63, CD151) of the PM and CD151 knockdown in cells blocked HPV16 entry [120]. This mode of endocytosis is distinct from other dynaminindependent routes in that, it is not inhibited by perturbation of cholesterol levels in the cell [120], nor does it rely on cellular levels of clathrin, caveolin, and flotillin. However, exact steps in this endocytic mechanism, as well as the associated molecular players, are still unclear. Recently, it was demonstrated that endocytosis of a tetraspanin, CD82, is dynamin-independent and occurs via 
a Cdc42-dependent GEEC-like pinocytic pathway [159]. It raises an interesting possibility that while tetraspanins regulate a distinct cholesterol-independent internalization route, they may utilize a different endocytic pathway for their own cellular entry [159].

Another such example, where inhibition of dynamin and cholesterol depletion does not affect uptake is the antagonist-induced internalization of nicotinic acetylcholine receptor [160]. Binding of the antagonist $\alpha$ bungarotoxin causes slow internalization of $\mathrm{nAChR}$, which proceeds via activation of Rac1 GTPase; inhibition of Rac1 activity or actin polymerization impairs the process [135]. These observations suggest that specialized protein-centric molecular platforms could assemble in the membrane to aid in this form of endocytosis.

There are now numerous examples of dynamin-independent endocytic systems $[9,114,161,162]$ that offer ample variety in terms of molecules employed and cargo endocytosed. Further study will enrich our understanding of principles behind mechanisms of endocytosis.

\section{Endocytic systems across phyla}

Undoubtedly, there are a variety of endocytic pathways available at the PM. Based on the environmental adaptations and molecular divergence, different kingdoms seem to have evolved specializations in endocytosis. In prokaryotes, evidence for a well-defined endocytic system is missing. A recent report using cryoelectron tomography showed various stages of the potential formation of membrane buds, including magnetosomes with inner membrane in Magnetospirillum magneticum [163]. These structures are reminiscent of endocytic membrane buds in higher organisms [163]. In unicellular eukaryotes, some subgroups have been shown to have spatially defined distribution of endocytic proteins, and specialized structures for endocytosis. For example, in Paramecium, endocytosis is confined to the cytopharynx, in Trypanosoma, assembly of endocytic machinery is restricted to flagellar pocket and in euglenoids, the ingestion tubule serves as the site of endocytosis [164].

In unicellular eukaryotes such as fungi, owing to the availability of genetic, pharmacological and biochemical tools, endocytosis (especially in the budding yeast, Saccharomyces cerevisiae) has been dissected in great detail [128], as discussed earlier. Riezman and colleagues had identified several endocytic mutants (end mutants) that were defective in internalization of the $\alpha$-factor [165]. Emr and colleagues had isolated dim (defective in internalization) mutants, defective in internalizing FM464 dye [166]. A quantitative genome-wide screen performed in yeast recently, examined the internalization of the VAMP homologue, Snc1 [167]. The screen uncovered novel regulators of endocytosis. It also uncovered phenotypes for predicted endocytosis genes for which no defects had previously been reported.

Apart from these fairly extensive studies in $S$. cerevisiae, initial ultrastructural studies in Neurospora and membrane dye uptake assays in Pisolithus tinctorius yielded no recognizable endocytic structures [168]. However, different species of Ustilago were found to contain homologs of endocytic proteins in their genome and could internalize lucifer yellow and the membrane marker FM4-64, suggesting the operation of endocytic pathways. These studies were carried out in cells after removal of the cell wall, and were therefore subject to validation in hyphae with an intact cell wall [169]. Similarly in Aspergillus nidulans, a cluster of vesicular structures at the tip of hyphae called Spitzenkörper were identified [170]. There seem to be differences in endocytic machinery in filamentous fungi and budding yeast. For example, internalization relies on ARF6 activity in A. nidulans, whereas in yeast, ARF6 is involved in polarity and bud growth but not in endocytosis $[170,171]$.

In invertebrates, Drosophila has been most extensively used to study endocytosis. A limited variety of endocytic routes are reported to operate in Drosophila, typically demonstrated in cultured hemocytes, isolated wing disc, oocyte nurse cells, and cultured neurons and pericardial cells for selected cell-surface proteins, primarily signaling receptors and bulk volume components [123, 172, 173]. Recently, increased usage of cultured cell lines derived from Drosophila, and advancement in techniques of genetic manipulation using dsRNA, have made it feasible to study endocytosis and other membrane-trafficking processes on a genome-wide scale [85, 174].

In nematodes, typified by $C$. elegans, a plethora of genes involved in yolk protein endocytosis and trafficking were uncovered in a genome-wide screen [175]. The function of these genes was further validated in mammalian cells, where mutation in some of these genes replicated transport defects as were observed in C. elegans. These studies also indicated that components of the molecular machinery underlying membrane trafficking may be conserved across systems. In a study that carried out extensive phylogenetic analysis of caveolin genes in metazoan (which provided evidence of extensive gene duplication), it was observed that vertebrate caveolin-1 and caveolin-3 isoforms and an invertebrate caveolin (Apis mellifera) were able to form morphologically identical caveolae in caveolin-1 null mouse cells, while the C. elegans caveolin could not. This indicates diversity of function in the caveolin gene family [156].

Primary and immortalized mammalian cell lines have 
proven to be an invaluable system to study endocytosis. While some internalization routes seem to be conserved in primary and immortalized cell lines, it is not certain whether their kinetics is conserved and if immortalization has consequences on overall endocytic activity of a cell type. Genetic knockout of known endocytic proteins in mice allows for the derivation of primary fibroblasts that are now routinely used to dissect the process [86]. These fibroblasts serve as excellent tools to study the involvement of candidate proteins in endocytosis - this approach not only avoids complexities associated with overexpression of their dominant-negative isoforms and off-target effects, but also can reflect on the functional redundancy of a given protein in the system.

In plants, taking into account the cell wall as a physical barrier to PM accessibility, and the high intracellular vacuolar pressure that builds up turgor pressure, the very existence of endocytosis was debated for a long time. In a study using electron microscopy, PM-associated invaginations containing electron-dense tracer and clathrin coats were visualized [176]. More recently, uptake of FM dyes was demonstrated in plant cells [177], following which many studies reported endocytosis of membrane markers and specific proteins in plant root hair cells, pollen tube, and cultured tobacco cells [178]. Now there is accumulating evidence about the diversity of endosomal pathways and endocytic compartments in plants. Although most of the studies used FM dyes to monitor internalization in plants, advancements in probing techniques are revealing a diversity of endocytic routes in this kingdom. Apart from clathrin-dependent receptor-mediated endocytosis, actin-sensitive fluidphase endocytosis in inner cortex cells of maize root [179] and PI3K-dependent bulk phase sucrose endocytosis in suspension culture cells of sycamore have been reported [180]. Blockade of clathrin-mediated endocytosis using ikarugamycin (Ika) did not inhibit uptake of positively charged nanogold particles, suggesting the operation of a clathrin-independent pathway [181]. However, in another study, Dhonukshe et al., showed that total membrane uptake in BY-2 tobacco cells could be inhibited by inhibiting clathrin using clathrin hub dominant negative form, suggesting that clathrin is absolutely required for any form of endocytosis [182]. It is possible that a diversity of endocytic portals are conserved in plant and animal systems, and several modes of endocytosis operate in plant cells in a cell type- and tissue- specific manner.

\section{Perspective}

Given the diversity and complexity of endocytic routes available in different cellular context, we provide here only a simplified overview of these pathways. Detailed understanding of the functioning of an individual pathway is complex in terms of its associated molecules and mechanism, and will require an individual focus.

It is likely that there are many different biochemical and physical principles behind the formation of vesicles in cell membrane. For example, cell-surface proteins such as the GPI-APs, which lack cytosolic extensions, cannot directly associate with cytosolic coat and adaptor proteins. These proteins therefore need to utilize radically different principles for sorting, membrane deformation, and vesicle generation at PM. Similarly, much less is known about endocytosis of membrane lipids. Dissection of the endocytic process of lipids will give insights into a novel way of vesicle formation. Also considering the multiplicity of functions of proteins, it will be interesting to investigate the overlapping functions of molecules known to be associated with specific trafficking pathways.

The identification of an endocytic pathway as distinct has been primarily based on associated cargo proteins or lipids, and molecular regulators; the contribution of kinetics and detailed physical mechanism to such categorization is not generally available except in some wellcharacterized situations, namely clathrin-pit endocytosis or endocytosis by actin-dependent forces in yeast. Considering the limited knowledge and vast diversity in molecular players that are employed for efficient functioning of endocytic trafficking pathways, it is difficult to clearly demarcate these mechanisms from one another.

On a cautionary note, it should be recognized that numerous cell lines have been utilized to study different types of endocytosis. These studies have helped uncover complex endocytic networks in a given cell system. The extent to which observations in one cell system may be extrapolated to another, or whether basic components of specific endocytic pathway are conserved across cell lines, is not completely clear. The possibility that modes of immortalization of cell lines itself could have consequences on how the endocytic pathways are configured in these cells has not been addressed.

Extensive work has been carried out to understand and elucidate the mechanism of endocytosis in some eukaryotes, and a considerable amount of information is available in some free-living eukaryotes and pathogens [183]. The integration of known information from complex endocytic systems in metazoan and comparison with uptake mechanisms in other eukaryotes could provide a means of understanding the scope of evolution, conservation, and redundancy in the internalization process across phyla.

Furthermore, whether specific endocytic modes are 
conserved in their entirety across phyla is also a subject of lively debate [183]. What is clear is that cells have many ways to endocytose material from the extracellular milieu, and perhaps as many reasons to do it by a particular mode. Until we understand the basic principles behind cargo concentration, membrane deformation, and scission in each of these modes it will be difficult to provide any rationale for the proliferation of the mechanisms of entry.

Regardless, the endocytic process, in addition to its role in uptake of nutrients and fluid, influences diverse key processes in metazoans, such as establishment of cellular asymmetry and modulation of signaling. In addition to its physiological roles, the endocytic process is also exploited by various pathogens. Insights into the underlying mechanisms will reveal new targets for drug design, and selective employment of these entry routes for drug delivery will also allow for the manipulation of cellular endocytic pathways to alleviate effects of specific diseases.

\section{References}

1 Swanson JA. Shaping cups into phagosomes and macropinosomes. Nat Rev Mol Cell Biol 2008; 9:639-649.

2 Groves E, Dart AE, Covarelli V, Caron E. Molecular mechanisms of phagocytic uptake in mammalian cells. Cell Mol Life Sci 2008; 65:1957-1976.

3 Kinchen JM, Ravichandran KS. Phagosome maturation: going through the acid test. Nat Rev Mol Cell Biol 2008; 9:781-795.

4 Huynh KK, Kay JG, Stow JL, Grinstein S. Fusion, fission, and secretion during phagocytosis. Physiology (Bethesda) 2007; 22:366-372.

5 Haas A. The phagosome: compartment with a license to kill. Traffic 2007; 8:311-330.

6 Kerr MC, Teasdale RD. Defining macropinocytosis. Traffic 2009; 10:364-371.

7 Falcone S, Cocucci E, Podini P, Kirchhausen T, Clementi E, Meldolesi J. Macropinocytosis: regulated coordination of endocytic and exocytic membrane traffic events. J Cell Sci 2006; 119(Pt 22):4758-4769.

8 Hansen CG, Bright NA, Howard G, Nichols BJ. SDPR induces membrane curvature and functions in the formation of caveolae. Nat Cell Biol 2009; 11:807-814.

9 Doherty GJ, McMahon HT. Mechanisms of endocytosis. Annu Rev Biochem 2009; 78:857-902

10 Bianco C, Griffin FM Jr, Silverstein SC. Studies of the macrophage complement receptor. Alteration of receptor function upon macrophage activation. J Exp Med 1975; 141:1278-1290.

11 Wright SD, Meyer BC. Phorbol esters cause sequential activation and deactivation of complement receptors on polymorphonuclear leukocytes. J Immunol 1986; 136:1759-1764.

12 Caron E, Hall A. Identification of two distinct mechanisms of phagocytosis controlled by different Rho GTPases. Science 1998; 282:1717-1721.

13 Allen LA, Aderem A. Molecular definition of distinct cytoskel- etal structures involved in complement- and Fc receptor-mediated phagocytosis in macrophages. J Exp Med 1996; 184:627637.

14 Patel JC, Galan JE. Differential activation and function of Rho GTPases during Salmonella-host cell interactions. J Cell Biol 2006; 175:453-463.

15 Pizarro-Cerda J, Cossart P. Subversion of cellular functions by Listeria monocytogenes. J Pathol 2006; 208:215-223.

16 Beemiller P, Hoppe AD, Swanson JA. A phosphatidylinositol3-kinase-dependent signal transition regulates ARF1 and ARF6 during Fcgamma receptor-mediated phagocytosis. PLoS Biol 2006; 4:e162.

17 Hoppe AD, Swanson JA. Cdc42, Rac1, and Rac2 display distinct patterns of activation during phagocytosis. Mol Biol Cell 2004; 15:3509-3519.

18 Park H, Cox D. Cdc42 regulates Fc \{gamma\} receptor-mediated phagocytosis through the activation and phosphorylation of WASP and N-WASP. Mol Biol Cell 2009; 20:4500-4508.

19 Bittner MA, Holz RW. Phosphatidylinositol-4,5-bisphosphate: actin dynamics and the regulation of ATP-dependent and -independent secretion. Mol Pharmacol 2005; 67:1089-1098.

20 Scott CC, Dobson W, Botelho RJ, et al. Phosphatidylinositol4,5-bisphosphate hydrolysis directs actin remodeling during phagocytosis. J Cell Biol 2005; 169:139-149.

21 Cox D, Tseng CC, Bjekic G, Greenberg S. A requirement for phosphatidylinositol 3-kinase in pseudopod extension. $J$ Biol Chem 1999; 274:1240-1247.

22 Braun V, Deschamps C, Raposo G, et al. AP-1 and ARF1 control endosomal dynamics at sites of FcR mediated phagocytosis. Mol Biol Cell 2007; 18:4921-4931.

23 Garin J, Diez R, Kieffer S, et al. The phagosome proteome: insight into phagosome functions. J Cell Biol 2001; 152:165180.

24 Policard A, Bessis M. [Pinocytosis (Lewis' phenomenon and its history).] Rev Hematol 1959; 14:487-495.

25 West MA, Bretscher MS, Watts C. Distinct endocytotic pathways in epidermal growth factor-stimulated human carcinoma A431 cells. J Cell Biol 1989; 109(6 Pt 1):2731-2739.

26 Mercanti V, Charette SJ, Bennett N, Ryckewaert JJ, Letourneur $\mathrm{F}$, Cosson P. Selective membrane exclusion in phagocytic and macropinocytic cups. J Cell Sci 2006; 119(Pt 19):4079-4087.

27 Swanson JA. Phorbol esters stimulate macropinocytosis and solute flow through macrophages. J Cell Sci 1989; 94(Pt 1):135-142.

28 Keller HU. Diacylglycerols and PMA are particularly effective stimulators of fluid pinocytosis in human neutrophils. J Cell Physiol 1990; 145:465-471.

29 Mercer J, Helenius A. Virus entry by macropinocytosis. Nat Cell Biol 2009; 11:510-520.

30 Sallusto F, Cella M, Danieli C, Lanzavecchia A. Dendritic cells use macropinocytosis and the mannose receptor to concentrate macromolecules in the major histocompatibility complex class II compartment: downregulation by cytokines and bacterial products. J Exp Med 1995; 182:389-400.

31 Ammendolia MG, Bertuccini L, Minelli F, Meschini S, Baldassarri L. A Sphingomonas bacterium interacting with epithelial cells. Res Microbiol 2004; 155:636-646.

32 Liu NQ, Lossinsky AS, Popik W, et al. Human immunodeficiency virus type 1 enters brain microvascular endothelia by 
macropinocytosis dependent on lipid rafts and the mitogenactivated protein kinase signaling pathway. J Virol 2002; 76:6689-6700.

33 Hewlett LJ, Prescott AR, Watts C. The coated pit and macropinocytic pathways serve distinct endosome populations. J Cell Biol 1994; 124:689-703.

34 Garrett WS, Chen LM, Kroschewski R, et al. Developmental control of endocytosis in dendritic cells by Cdc42. Cell 2000; 102:325-334.

35 Dharmawardhane S, Schürmann A, Sells MA, Chernoff J, Schmid SL, Bokoch GM. Regulation of macropinocytosis by p21-activated kinase-1. Mol Biol Cell 2000; 11:3341-3352.

36 Liberali P, Kakkonen E, Turacchio G, et al. The closure of Pak1-dependent macropinosomes requires the phosphorylation of CtBP1/BARS. EMBO J 2008; 27:970-981.

37 Cao H, Chen J, Awoniyi M, Henley JR, McNiven MA. Dynamin 2 mediates fluid-phase micropinocytosis in epithelial cells. J Cell Sci 2007; 120(Pt 23):4167-4177.

38 Liu YW, Surka MC, Schroeter T, Lukiyanchuk V, Schmid SL. Isoform and splice-variant specific functions of dynamin-2 revealed by analysis of conditional knock-out cells. Mol Biol Cell 2008; 19:5347-5359.

39 Schlunck G, Damke H, Kiosses WB, et al. Modulation of Rac localization and function by dynamin. Mol Biol Cell 2004; 15:256-267.

40 Yeung T, Gilbert GE, Shi J, Silvius J, Kapus A, Grinstein S. Membrane phosphatidylserine regulates surface charge and protein localization. Science 2008; 319:210-213.

41 Sarasij RC, Mayor S, Rao M. Chirality-induced budding: a raft-mediated mechanism for endocytosis and morphology of caveolae? Biophys J 2007; 92:3140-3158.

42 Pearse BM. Clathrin: a unique protein associated with intracellular transfer of membrane by coated vesicles. Proc Natl Acad Sci USA 1976; 73:1255-1259.

43 Roth TF, Porter KR. Yolk protein uptake in the oocyte of the mosquito Aedes Aegypti. L. J Cell Biol 1964; 20:313-332.

44 Kirchhausen, T, Clathrin. Annu Rev Biochem 2000; 69:699727.

45 Marsh M, McMahon HT. The structural era of endocytosis. Science 1999; 285:215-220.

46 Ehrlich M, Boll W, Van Oijen A, et al. Endocytosis by random initiation and stabilization of clathrin-coated pits. Cell 2004; 118:591-605.

47 Ungewickell EJ, Hinrichsen L. Endocytosis: clathrin-mediated membrane budding. Curr Opin Cell Biol 2007; 19:417-425.

48 Merrifield CJ, Perrais D, Zenisek D. Coupling between clathrin-coated-pit invagination, cortactin recruitment, and membrane scission observed in live cells. Cell 2005; 121:593-606.

49 Young A. Structural insights into the clathrin coat. Semin Cell Dev Biol 2007; 18:448-458.

50 Kirchhausen T. Adaptors for clathrin-mediated traffic. Annu Rev Cell Dev Biol 1999; 15:705-732.

51 Habermann B. The BAR-domain family of proteins: a case of bending and binding? EMBO Rep 2004; 5:250-255.

52 Blood PD, Voth GA. Direct observation of Bin/amphiphysin/ Rvs (BAR) domain-induced membrane curvature by means of molecular dynamics simulations. Proc Natl Acad Sci USA 2006; 103:15068-15072.

53 Kanaseki T, Kadota K. The 'vesicle in a basket'. A morpholog- ical study of the coated vesicle isolated from the nerve endings of the guinea pig brain, with special reference to the mechanism of membrane movements. J Cell Biol 1969; 42:202-220.

54 Puthenveedu MA, von Zastrow M. Cargo regulates clathrincoated pit dynamics. Cell 2006; 127:113-124.

55 Wolfe BL, Trejo J. Clathrin-dependent mechanisms of G protein-coupled receptor endocytosis. Traffic 2007; 8:462-470.

56 Benmerah A, Lamaze C. Clathrin-coated pits: vive la difference? Traffic 2007; 8:970-982.

57 Carpenter G, Cohen S. Epidermal growth factor. Annu Rev Biochem 1979; 48:193-216.

58 Sigismund S, Woelk T, Puri C, et al. Clathrin-independent endocytosis of ubiquitinated cargos. Proc Natl Acad Sci USA 2005; 102:2760-2765.

59 Marsh M, Helenius A. Virus entry: open sesame. Cell 2006; 124:729-740.

60 Rust MJ, Lakadamyali M, Zhang F, Zhuang X. Assembly of endocytic machinery around individual influenza viruses during viral entry. Nat Struct Mol Biol 2004; 11:567-573.

61 Veiga E, Cossart P. Listeria hijacks the clathrin-dependent endocytic machinery to invade mammalian cells. Nat Cell Biol 2005; 7:894-900

62 Palade GE. Fine structure of blood capillaries. J Appl Phys 1953; 24:1424.

63 Rothberg KG, Heuser JE, Donzell WC, Ying YS, Glenney JR, Anderson RG. Caveolin, a protein component of caveolae membrane coats. Cell 1992; 68:673-682.

64 Chadda R, Howes MT, Plowman SJ, Hancock JF, Parton RG, Mayor S. Cholesterol-sensitive $\mathrm{Cdc} 42$ activation regulates actin polymerization for endocytosis via the GEEC pathway. Traffic 2007; 8:702-717.

65 Hill MM, Bastiani M, Luetterforst R, et al. PTRF-Cavin, a conserved cytoplasmic protein required for caveola formation and function. Cell 2008; 132:113-24.

66 Mirre C, Monlauzeur L, Garcia M, Delgrossi MH, Le Bivic A. Detergent-resistant membrane microdomains from Caco2 cells do not contain caveolin. Am J Physiol 1996; 271(3 Pt 1):C887-894.

67 Vogel U, Sandvig K, van Deurs B. Expression of caveolin-1 and polarized formation of invaginated caveolae in Caco-2 and MDCK II cells. J Cell Sci 1998; 111(Pt 6):825-832.

68 Fra AM, Williamson E, Simons K, Parton RG. De novo formation of caveolae in lymphocytes by expression of VIP21caveolin. Proc Natl Acad Sci USA 1995; 92:8655-8659.

69 McMahon HT, Gallop JL. Membrane curvature and mechanisms of dynamic cell membrane remodelling. Nature 2005; 438:590-596.

70 Kalia M, Kumari S, Chadda R, Hill MM, Parton RG, Mayor S. Arf6-independent GPI-anchored protein-enriched early endosomal compartments fuse with sorting endosomes via a Rab5/ phosphatidylinositol-3'-kinase-dependent machinery. Mol Biol Cell 2006; 17:3689-3704.

71 Chadda R, Mayor S. PTRF triggers a cave in. Cell 2008; 132:23-24.

72 Schnitzer JE, Oh P, Pinney E, Allard J. Filipin-sensitive caveolae-mediated transport in endothelium: reduced transcytosis, scavenger endocytosis, and capillary permeability of select macromolecules. J Cell Biol 1994; 127:1217-1232.

73 Benlimame N, Le PU, Nabi IR. Localization of autocrine 
motility factor receptor to caveolae and clathrin-independent internalization of its ligand to smooth endoplasmic reticulum. Mol Biol Cell 1998; 9:1773-1786.

74 Herreros J, Ng T, Schiavo G. Lipid rafts act as specialized domains for tetanus toxin binding and internalization into neurons. Mol Biol Cell 2001; 12:2947-2960.

75 Shogomori H, Futerman AH. Cholera toxin is found in detergent-insoluble rafts/domains at the cell surface of hippocampal neurons but is internalized via a raft-independent mechanism. J Biol Chem 2001; 276:9182-9188.

76 Anderson HA, Chen Y, Norkin LC. Bound simian virus 40 translocates to caveolin-enriched membrane domains, and its entry is inhibited by drugs that selectively disrupt caveolae. Mol Biol Cell 1996; 7:1825-1834.

77 Pelkmans L, Helenius A. Endocytosis via caveolae. Traffic 2002; 3:311-320.

78 Thomsen P, Roepstorff K, Stahlhut M, van Deurs B. Caveolae are highly immobile plasma membrane microdomains, which are not involved in constitutive endocytic trafficking. Mol Biol Cell 2002; 13:238-250.

79 Pelkmans L, Kartenbeck J, Helenius A. Caveolar endocytosis of simian virus 40 reveals a new two-step vesicular-transport pathway to the ER. Nat Cell Biol 2001; 3:473-483.

80 Singh RD, Puri V, Valiyaveettil JT, Marks DL, Bittman R, Pagano RE. Selective caveolin-1-dependent endocytosis of glycosphingolipids. Mol Biol Cell 2003; 14:3254-3265.

81 Sharma DK, Brown JC, Cheng Z, Holicky EL, Marks DL, Pagano RE. The glycosphingolipid, lactosylceramide, regulates beta1-integrin clustering and endocytosis. Cancer Res 2005; 65:8233-8241.

82 Sharma DK, Brown JC, Choudhury A, et al. Selective stimulation of caveolar endocytosis by glycosphingolipids and cholesterol. Mol Biol Cell 2004; 15:3114-3122.

83 del Pozo MA, Balasubramanian N, Alderson NB, et al. Phospho-caveolin-1 mediates integrin-regulated membrane domain internalization. Nat Cell Biol 2005; 7:901-908.

84 del Pozo MA, Alderson NB, Kiosses WB, Chiang HH, Anderson RG, Schwartz MA. Integrins regulate Rac targeting by internalization of membrane domains. Science 2004; 303:839842.

85 Agaisse H, Burrack LS, Philips JA, Rubin EJ, Perrimon N, Higgins DE. Genome-wide RNAi screen for host factors required for intracellular bacterial infection. Science 2005; 309:1248-1251.

86 Razani B, Engelman JA, Wang XB, et al. Caveolin-1 null mice are viable but show evidence of hyperproliferative and vascular abnormalities. J Biol Chem 2001; 276:38121-38138.

87 Damm EM, Pelkmans L, Kartenbeck J, Mezzacasa A, Kurzchalia T, Helenius A. Clathrin- and caveolin-1-independent endocytosis: entry of simian virus 40 into cells devoid of caveolae. J Cell Biol 2005; 168:477-488.

88 Kirkham M, Fujita A, Chadda R, et al. Ultrastructural identification of uncoated caveolin-independent early endocytic vehicles. J Cell Biol 2005; 168:465-476.

89 Hernández-Deviez DJ, Howes MT, Laval SH, Bushby K, Hancock JF, Parton RG. Caveolin regulates endocytosis of the muscle repair protein, dysferlin. J Biol Chem 2008; 283:64766488.

90 Lajoie P, Kojic LD, Nim S, Li L, Dennis JW, Nabi IR. Ca- veolin-1 regulation of dynamin-dependent, raft-mediated endocytosis of cholera toxin b-subunit occurs independently of caveolae. J Cell Mol Med 2009 Mar 6. doi:10.1111/j.15824934.2009.00732.x

91 Nevins AK, Thurmond DC, Caveolin-1 functions as a novel $\mathrm{Cdc} 42$ guanine nucleotide dissociation inhibitor in pancreatic beta-cells. J Biol Chem 2006; 281:18961-18972.

92 Koenig JH, Ikeda K. Disappearance and reformation of synaptic vesicle membrane upon transmitter release observed under reversible blockage of membrane retrieval. J Neurosci 1989; 9:3844-3860.

93 Kosaka T, Ikeda K. Reversible blockage of membrane retrieval and endocytosis in the garland cell of the temperature-sensitive mutant of Drosophila melanogaster, shibire ${ }^{t s I}$. J Cell Biol 1983; 97:499-507.

94 van der Bliek AM, Redelmeier TE, Damke H, Tisdale EJ, Meyerowitz EM, Schmid SL. Mutations in human dynamin block an intermediate stage in coated vesicle formation. $J$ Cell Biol 1993; 122:553-563.

95 Cao H, Garcia F, McNiven MA. Differential distribution of dynamin isoforms in mammalian cells. Mol Biol Cell 1998; 9:2595-2609.

96 Shpetner HS, Vallee RB. Identification of dynamin, a novel mechanochemical enzyme that mediates interactions between microtubules. Cell 1989; 59:421-432.

97 Chen MS, Obar RA, Schroeder CC, et al. Multiple forms of dynamin are encoded by shibire, a Drosophila gene involved in endocytosis. Nature 1991; 351:583-586.

98 Damke H, Baba T, Warnock DE, Schmid SL. Induction of mutant dynamin specifically blocks endocytic coated vesicle formation. J Cell Biol 1994; 127:915-934.

99 Takei K, McPherson PS, Schmid SL, De Camilli P. Tubular membrane invaginations coated by dynamin rings are induced by GTP-gamma S in nerve terminals. Nature 1995; 374:186190.

100Slepnev VI, Ochoa GC, Butler MH, De Camilli P. Tandem arrangement of the clathrin and AP-2 binding domains in amphiphysin 1 and disruption of clathrin coat function by amphiphysin fragments comprising these sites. J Biol Chem 2000; 275:17583-17589.

101 Takei K, Yoshida Y, Yamada H. Regulatory mechanisms of dynamin-dependent endocytosis. J Biochem 2005; 137:243247.

102 Thompson HM, and McNiven MA. Dynamin: switch or pinchase? Curr Biol 2001; 11:R850.

103Roux A, Uyhazi K, Frost A, De Camilli P. GTP-dependent twisting of dynamin implicates constriction and tension in membrane fission. Nature 2006; 441:528-531.

104Pucadyil TJ, Schmid SL. Real-time visualization of dynamincatalyzed membrane fission and vesicle release. Cell 2008; 135:1263-1275.

105 Bashkirov PV, Akimov SA, Evseev AI, Schmid SL, Zimmerberg J, Frolov VA. GTPase cycle of dynamin is coupled to membrane squeeze and release, leading to spontaneous fission. Cell 2008; 135:1276-1286.

106Roux A, Antonny B, The long and short of membrane fission. Cell 2008; 135:3.

107Henley JR, Krueger EW, Oswald BJ, McNiven MA. Dynaminmediated internalization of caveolae. J Cell Biol 1998; 141:85- 
99.

108Kolpak AL, Jiang J, Guo D, et al. Negative guidance factorinduced macropinocytosis in the growth cone plays a critical role in repulsive axon turning. $J$ Neurosci 2009; 29:1048810498.

109Lamaze C, Dujeancourt A, Baba T, Lo CG, Benmerah A, Dautry-Varsat A. Interleukin 2 receptors and detergent-resistant membrane domains define a clathrin-independent endocytic pathway. Mol Cell 2001; 7:661-671.

110 Saavedra L, Mohamed A, Ma V, Kar S, de Chaves EP. Internalization of beta-amyloid peptide by primary neurons in the absence of apolipoprotein E. J Biol Chem 2007; 282:3572235732 .

111 Orth JD, Krueger EW, Weller SG, McNiven MA. A novel endocytic mechanism of epidermal growth factor receptor sequestration and internalization. Cancer Res 2006; 66:36033610 .

112 Doxsey SJ, Brodsky FM, Blank GS, Helenius A. Inhibition of endocytosis by anti-clathrin antibodies. Cell 1987; 50:453-463.

113 Moya M, Dautry-Varsat A, Goud B, Louvard D, Boquet P. Inhibition of coated pit formation in Hep2 cells blocks the cytotoxicity of diphtheria toxin but not that of ricin toxin. $J$ Cell Biol 1985; 101:548-559.

114 Conner SD, Schmid SL. Regulated portals of entry into the cell. Nature 2003; 422:37-44.

115 Cheng ZJ, Singh RD, Sharma DK, et al. Distinct mechanisms of clathrin-independent endocytosis have unique sphingolipid requirements. Mol Biol Cell 2006; 17:3197-3210.

116Zoncu R, Perera RM, Sebastian R, et al. Loss of endocytic clathrin-coated pits upon acute depletion of phosphatidylinositol 4,5-bisphosphate. Proc Natl Acad Sci USA 2007; 104:37933798 .

117 Römer W, Berland L, Chambon V, et al. Shiga toxin induces tubular membrane invaginations for its uptake into cells. $\mathrm{Na}$ ture 2007; 450:670-675.

118 Sabharanjak S, Mayor S. Folate receptor endocytosis and trafficking. Adv Drug Deliv Rev 2004; 56:1099-1109.

119 Sabharanjak S, Sharma P, Parton RG, Mayor S. GPI-anchored proteins are delivered to recycling endosomes via a distinct cdc42-regulated, clathrin-independent pinocytic pathway. Dev Cell 2002; 2:411-423.

120 Spoden G, Freitag K, Husmann M, et al. Clathrin- and caveolin-independent entry of human papillomavirus type $16-$ involvement of tetraspanin-enriched microdomains (TEMs). PLoS ONE 2008; 3:e3313.

121 Faire K, Trent F, Tepper JM, Bonder EM. Analysis of dynamin isoforms in mammalian brain: dynamin-1 expression is spatially and temporally regulated during postnatal development. Proc Natl Acad Sci USA 1992; 89:8376-8380.

122 Cook T, Mesa K, Urrutia R. Three dynamin-encoding genes are differentially expressed in developing rat brain. $J$ Neurochem 1996; 67:927-931.

123 Guha A, Sriram V, Krishnan KS, Mayor S. Shibire mutations reveal distinct dynamin-independent and -dependent endocytic pathways in primary cultures of Drosophila hemocytes. J Cell Sci 2003; 116(Pt 16):3373-3386.

124Grant D, Unadkat S, Katzen A, Krishnan KS, Ramaswami M. Probable mechanisms underlying interallelic complementation and temperature-sensitivity of mutations at the shibire locus of
Drosophila melanogaster. Genetics 1998; 149:1019-1030.

125 Grassart A, Dujeancourt A, Lazarow PB, Dautry-Varsat A, Sauvonnet N. Clathrin-independent endocytosis used by the IL-2 receptor is regulated by Rac1, Pak1 and Pak2. EMBO Rep 2008; 9:356-362.

126 Galletta BJ, Cooper JA. Actin and endocytosis: mechanisms and phylogeny. Curr Opin Cell Biol 2009; 21:20-27.

127Munn AL, Stevenson BJ, Geli MI, Riezman H. end5, end6, and end7: mutations that cause actin delocalization and block the internalization step of endocytosis in Saccharomyces cerevisiae. Mol Biol Cell 1995; 6:1721-1742.

128 Toret CP, Drubin DG. The budding yeast endocytic pathway. $J$ Cell Sci 2006; 119(Pt 22):4585-4587.

129 Kaksonen M, Sun Y, Drubin DG. A pathway for association of receptors, adaptors, and actin during endocytic internalization. Cell 2003; 115:475-487.

130Kaksonen M, Toret CP, Drubin DG. A modular design for the clathrin- and actin-mediated endocytosis machinery. Cell 2005; 123:305-320.

131 Liu J, Kaksonen M, Drubin DG, Oster G. Endocytic vesicle scission by lipid phase boundary forces. Proc Natl Acad Sci USA 2006; 103:10277-10282.

132 Geli MI, Riezman H. Role of type I myosins in receptor-mediated endocytosis in yeast. Science 1996; 272:533-535.

133 Girao H, Geli MI, Idrissi FZ. Actin in the endocytic pathway: from yeast to mammals. FEBS Lett 2008; 582:2112-2119.

134Kaksonen M, Peng HB, Rauvala H. Association of cortactin with dynamic actin in lamellipodia and on endosomal vesicles. J Cell Sci 2000; 113(Pt 24):4421-4426.

135 Kumari S, Borroni V, Chaudhry A, et al. Nicotinic acetylcholine receptor is internalized via a Rac-dependent, dynaminindependent endocytic pathway. J Cell Biol 2008; 181:11791193.

136Sokac AM, Co C, Taunton J, Bement W. Cdc42-dependent actin polymerization during compensatory endocytosis in $\mathrm{Xe}$ nopus eggs. Nat Cell Biol 2003; 5:727-732.

137 Sharma P, Varma R, Sarasij RC, et al. Nanoscale organization of multiple GPI-anchored proteins in living cell membranes. Cell 2004; 116:577-589.

138Bhagatji P, Leventis R, Comeau J, Refaei M, Silvius JR. Steric and not structure-specific factors dictate the endocytic mechanism of glycosylphosphatidylinositol-anchored proteins. J Cell Biol 2009; 186:615-628.

139Lundmark R, Doherty GJ, Howes MT, et al. The GTPase-activating protein GRAF1 regulates the CLIC/GEEC endocytic pathway. Curr Biol 2008; 18:1802-1808.

140Ritter TE, Fajardo O, Matsue H, Anderson RG, Lacey SW. Folate receptors targeted to clathrin-coated pits cannot regulate vitamin uptake. Proc Natl Acad Sci USA 1995; 92:3824-3828.

141 Gauthier NC, Monzo P, Kaddai V, Doye A, Ricci V, Boquet P. Helicobacter pylori VacA cytotoxin: a probe for a clathrinindependent and Cdc42-dependent pinocytic pathway routed to late endosomes. Mol Biol Cell 2005; 16:4852-4866.

142 Yarar D, Waterman-Storer CM, Schmid SL. SNX9 couples actin assembly to phosphoinositide signals and is required for membrane remodeling during endocytosis. Dev Cell 2007; 13:43-56.

143 Naslavsky N, Weigert R, Donaldson JG. Convergence of nonclathrin- and clathrin-derived endosomes involves Arf6 inacti- 
vation and changes in phosphoinositides. Mol Biol Cell 2003; 14:417-431.

144Donaldson JG, Porat-Shliom N, Cohen LA. Clathrin-independent endocytosis: a unique platform for cell signaling and PM remodeling. Cell Signal 2009; 21:1-6.

145Radhakrishna H, Donaldson JG. ADP-ribosylation factor 6 regulates a novel plasma membrane recycling pathway. $J$ Cell Biol 1997; 139:49-61.

146Eyster CA, Higginson JD, Huebner R, et al. Discovery of new cargo proteins that enter cells through clathrin-independent endocytosis. Traffic 2009; 10:590-599.

147Nishi K, Saigo K. Cellular internalization of green fluorescent protein fused with herpes simplex virus protein VP22 via a lipid raft-mediated endocytic pathway independent of caveolae and Rho family GTPases but dependent on dynamin and Arf6. $J$ Biol Chem 2007; 282:15.

148Balasubramanian N, Scott D, Castle JD, Casanova JE, Schwartz MA. Arf6 and microtubules in adhesion-dependent trafficking of lipid rafts. Nat Cell Biol 2007; 9:11.

149Santy LC, Casanova JE. Activation of ARF6 by ARNO stimulates epithelial cell migration through downstream activation of both Rac1 and phospholipase D. J Cell Biol 2001; 154:599610 .

150 Arnaoutova I, Jackson CL, Al-Awar OS, Donaldson JG, Loh YP. Recycling of Raft-associated prohormone sorting receptor carboxypeptidase E requires interaction with ARF6. Mol Biol Cell 2003; 14:4448-4457.

151 Glebov OO, Bright NA, Nichols BJ. Flotillin-1 defines a clathrin-independent endocytic pathway in mammalian cells. Nat Cell Biol 2006; 8:46-54.

152Babuke T, Tikkanen R. Dissecting the molecular function of reggie/flotillin proteins. Eur J Cell Biol 2007; 86:525-532.

153Frick M, Bright NA, Riento K, Bray A, Merrified C, Nichols BJ. Coassembly of flotillins induces formation of membrane microdomains, membrane curvature, and vesicle budding. Curr Biol 2007; 17:1151-1156.

154 Volonte D, Galbiati F, Li S, Nishiyama K, Okamoto T, Lisanti MP. Flotillins/cavatellins are differentially expressed in cells and tissues and form a hetero-oligomeric complex with caveolins in vivo. Characterization and epitope-mapping of a novel flotillin-1 monoclonal antibody probe. J Biol Chem 1999; 274:12702-12709.

155Hansen CG, Nichols BJ. Molecular mechanisms of clathrinindependent endocytosis. J Cell Sci 2009; 122(Pt 11):17131721.

156Kirkham M, Nixon SJ, Howes MT, et al. Evolutionary analysis and molecular dissection of caveola biogenesis. J Cell Sci 2008; 121(Pt 12):2075-2086.

157 Giri B, Dixit VD, Ghosh MC, et al. CXCL12-induced partitioning of flotillin-1 with lipid rafts plays a role in CXCR4 function. Eur J Immunol 2007; 37:2104-2116.

158Riento K, Frick M, Schafer I, Nichols BJ. Endocytosis of flotillin-1 and flotillin-2 is regulated by Fyn kinase. J Cell Sci 2009; 122(Pt 7):912-918.

159Xu C, Zhang YH, Thangavel M, et al. CD82 endocytosis and cholesterol-dependent reorganization of tetraspanin webs and lipid rafts. FASEB J 2009; 23:3273-3288.

160Borroni V, Baier CJ, Lang T, et al. Cholesterol depletion activates rapid internalization of submicron-sized acetylcholine receptor domains at the cell membrane. Mol Membr Biol 2007; 24:1-15.

161 Mayor S, Pagano RE. Pathways of clathrin-independent endocytosis. Nat Rev Mol Cell Biol 2007; 8:603-612.

162 Sandvig K, Torgersen ML, Raa HA, van Deurs B. Clathrin-independent endocytosis: from nonexisting to an extreme degree of complexity. Histochem Cell Biol 2008; 129:267-276.

163 Komeili A, Li Z, Newman DK, Jensen GJ. Magnetosomes are cell membrane invaginations organized by the actin-like protein MamK. Science 2006; 311:242-245.

164Field MC, Gabernet-Castello C, Dacks JB. Reconstructing the evolution of the endocytic system: insights from genomics and molecular cell biology. Adv Exp Med Biol 2007; 607:84-96.

165D'Hondt K, Heese-Peck A, Riezman H. Protein and lipid requirements for endocytosis. Annu Rev Genet 2000; 34:255295.

166Wendland B, McCaffery JM, Xiao Q, Emr SD. A novel fluorescence-activated cell sorter-based screen for yeast endocytosis mutants identifies a yeast homologue of mammalian eps 15. J Cell Biol 1996; 135:16.

167Burston HE, Maldonado-Báez L, Davey M, et al. Regulators of yeast endocytosis identified by systematic quantitative analysis. J Cell Biol 2009; 185:1097-1110.

168Fuchs U, Steinberg G. Endocytosis in the plant-pathogenic fungus Ustilago maydis. Protoplasma 2005; 226:75-80.

169Steinberg G, Schliwa M, Lehmler C, Bölker M, Kahmann R, McIntosh JR. Kinesin from the plant pathogenic fungus Ustilago maydis is involved in vacuole formation and cytoplasmic migration. J Cell Sci 1998; 111( Pt 15):2235-2246.

170Lee SC, Schmidtke SN, Dangott LJ, Shaw BD. Aspergillus nidulans ArfB plays a role in endocytosis and polarized growth. Eukaryot Cell 2008; 7:1278-1288.

171 Huang CF, Liu YW, Tung L, Lin CH, Lee FJ. Role for Arf3p in development of polarity, but not endocytosis, in Saccharomyces cerevisiae. Mol Biol Cell 2003; 14:3834-3847.

172Fischer JA, Eun SH, Doolan BT. Endocytosis, endosome trafficking, and the regulation of Drosophila development. Annu Rev Cell Dev Biol 2006; 22:181-206.

173 Das D, Aradhya R, Ashoka D, Inamdar M. Macromolecular uptake in Drosophila pericardial cells requires rudhira function. Exp Cell Res 2008; 314:1804-1810.

174 Stroschein-Stevenson SL, Foley E, O'Farrell PH, Johnson AD. Phagocytosis of Candida albicans by RNAi-treated Drosophila S2 cells. Methods Mol Biol 2009; 470:347-358.

175Balklava Z, Pant S, Fares H, Grant BD. Genome-wide analysis identifies a general requirement for polarity proteins in endocytic traffic. Nat Cell Biol 2007; 9:1066-1073.

176 Saxton MJ, Breidenbach RW. Receptor-mediated endocytosis in plants is energetically possible. Plant Physiol 1988; 86:993995.

177Emans N, Zimmermann S, Fischer R. Uptake of a fluorescent marker in plant cells is sensitive to brefeldin A and wortmannin. Plant Cell 2002; 14:71-86.

178Müller J, Mettbach U, Menzel D, Samaj J. Molecular dissection of endosomal compartments in plants. Plant Physiol 2007; 145:293-304.

179Baluska F, Samaj J, Hlavacka A, Kendrick-Jones J, Volkmann D. Actin-dependent fluid-phase endocytosis in inner cortex cells of maize root apices. J Exp Bot 2004; 55:463-473. 
180Etxeberria E, Baroja-Fernandez E, Muñoz FJ, Pozueta-Romero J. Sucrose-inducible endocytosis as a mechanism for nutrient uptake in heterotrophic plant cells. Plant Cell Physiol 2005; 46:474-481.

181 Moscatelli A, Ciampolini F, Rodighiero S, et al. Distinct endocytic pathways identified in tobacco pollen tubes using charged nanogold. J Cell Sci 2007; 120(Pt 21):3804-3819.
182Dhonukshe P, Aniento F, Hwang I, et al. Clathrin-mediated constitutive endocytosis of PIN auxin efflux carriers in Arabidopsis. Curr Biol 2007; 17:520-527.

183 Dacks JB, Poon PP, Field MC. Phylogeny of endocytic components yields insight into the process of nonendosymbiotic organelle evolution. Proc Natl Acad Sci USA 2008; 105:588593. 